\title{
Efectividad analgésica del tapentadol en el dolor neuropático secundario al síndrome postlaminectomía
}

\section{Analgesic effectiveness of tapentadol in neuropathic pain secondary to post-laminectomy syndrome}

\author{
J. J. Medina Pérez ${ }^{1,2}$, G. A. Vega Rosas², M. E. Medina Pérez² y S. G. Coubert Pelayo²
}

${ }^{1}$ Clínica del Dolor, Centro Médico Nacional 20 de Noviembre ISSSTE. Ciudad de México, México. ${ }^{2} S e r v i c i o ~ d e$ Algología y Medicina del Dolor, Hospital Ángeles Mocel. Ciudad de México, México

\section{RESUMEN}

Objetivo: El objetivo del presente estudio es evaluar la eficacia y seguridad de tapentadol de liberación a 100 o 200 mg vía oral cada 24 horas, de acción prolongada, para el tratamiento del síndrome postlaminectomía (SPL) en una serie de pacientes con dolor neuropático en tratamiento analgésico inefectivo.

Material y métodos: Se realizó un estudio unicéntrico, longitudinal, prospectivo y observacional, en el que se reclutaron 30 pacientes a la clínica de dolor de un centro de referencia de tercer nivel que sufrían de SPL y que cumplían con todos los criterios de inclusión; a quienes se les aplicó el cuestionario Brief Pain Inventory en su versión en español y el test de Lanss antes y después de iniciar tratamiento con tapentadol, y se aplicó una $t$ de Student para comparar la efectividad global del tratamiento del dolor neuropático.

Resultado: Se analizaron datos de 30 pacientes, de los cuales 19 fueron mujeres (63,3\%) y 11 hombres [36,6 \%] con diagnóstico de SPL confirmado y con características de dolor de tipo neuropático, quienes fueron divididos en dos grupos: el primer grupo de 13 pacientes $(43,3 \%$ ) recibió tapentadol a $100 \mathrm{mg}$ vía oral cada 24 horas y el segundo de 17 pacientes [56,6 \%) recibió 200 mg vía oral cada 24 horas por cuatro semanas. Se les dio un seguimiento de 4 sema-

\section{ABSTRACT}

Objective: The objective of this study is to evaluate the efficacy and safety of long-acting tapentadol 100 or $200 \mathrm{mg}$ orally every 24 hours for the treatment of Postlaminectomy Syndrome (SPL) in a series of patients with pain neuropathic in ineffective analgesic treatment.

Material and methods: A single-center, longitudinal, prospective and observational study was conducted, in which 30 patients were recruited to the pain clinic of a third-level reference center who suffered from SPL and who met all the inclusion criteria; To whom the Brief Pain Inventory questionnaire in its Spanish version and the Lanss Test were applied before and after starting treatment with tapentadol and a student's $t$ was applied to compare the overall effectiveness of the treatment of neuropathic pain.

Results: Data from 30 patients were analyzed, of which 19 were women (63.3\%) and 11 were men [36.6\%) with a diagnosis of confirmed SPL and the presentation of neuropathic pain, who were divided into two groups. The first group of 13 patients (43.3\%) received tapentadol at $100 \mathrm{mg}$ orally every 24 hours, and the second group of 17 patients (56.6\%) received $200 \mathrm{mg}$ orally every 24 hours for four weeks. They were followed up for 4 weeks and statistically signifi-
Medina Pérez JJ, Vega Rosas GA, Medina Pérez ME, Coubert Pelayo SG. Efectividad analgésica del tapentadol en el dolor neuropático secundario al síndrome postlaminectomía. Rev Soc Esp Dolor. 2021;28(2):71-75
Recibido: 30-05-2020

Aceptado: 12-05-2021

Correspondencia: Andrés Vega Rosas andresvr315@gmail.com 
nas y se encontró una disminución estadísticamente significativa (valor de $p=0,05$ ) del dolor neuropático en la consulta subsecuente de la clínica del SPL.

Palabras clave: Dolor neuropático, dolor crónico, síndrome postlaminectomía, tapentadol, opioides, cirugía ortopédica. cant improvement ( $p$ value $=0.05$ ) was found in the SPL clinic.

Key words: Neuropathic pain, chronic pain, postlaminectomy syndrome, tapentadol, opioids, orthopedic surgery.

\section{INTRODUCCIÓN}

El síndrome postlaminectomía (SPL) es una entidad clínica compleja debido a que es un diagnóstico directamente relacionado a un tratamiento quirúrgico, y en la actualidad es de los principales motivos de consulta en las unidades de dolor. El SPL es resultado de tratamientos quirúrgicos que condujeron o dejaron al paciente con dolor crónico axial y/o dolor radicular de igual o mayor intensidad que el previo a la cirugía [1]. El dolor presenta una mala respuesta a los tratamientos convencionales y es de difícil control. En los Estados Unidos, donde la cirugía de columna excede las 300.000 operaciones al año, del 10 al $40 \%$ de las operaciones de columna lumbar resultan en SPL [2]; los datos estadísticos no difieren en gran medida del contexto mexicano.

De acuerdo con la Asociación Internacional para el Estudio del Dolor (IASP por sus siglas en inglés) el dolor neuropático (DN) es definido como "dolor causado por una lesión o disfunción del sistema nervioso somatosensorial" [3]. El DN se caracteriza por ser un dolor tipo urente, quemante, asociado a espasmos musculares, disminución de la fuerza, sensación de toques eléctricos y/o sensibilidad. Este dolor puede ser consecuencia de procesos inflamatorios en las raíces nerviosas y por sensibilización periférica. En todos los casos de DN debe recordarse que el dolor de espalda crónico representa una transformación del dolor nociceptivo a dolor neuropático que debe tomar en cuenta la percepción subyacente del paciente, ya que el SPL representa un reto clínico debido a la complejidad en su fisiopatología, y usualmente no responde a los analgésicos convencionales y sus combinaciones, además de que los síntomas secundarios asociados son desagradables para los pacientes y los llevan a interrumpir el tratamiento (4).

El tratamiento del dolor neuropático tiene su base en grupos de medicamentos diferentes a los analgésicos convencionales, por ejemplo, los anticonvulsivantes, antidepresivos tricíclicos y analgésicos puros, los opioides. El tapentadol es un opioide de acción central cuya indicación es en el manejo de dolor crónico severo, y que presenta dos mecanismos de acción, el agonismo parcial opioide en receptores $\mu$ y la inhibición de la recaptación de la noradrenalina al mismo tiempo. Se une a los receptores $\mu$-opioides selectivamente, de forma moderada, en comparación con morfina u oxicodona [5], ya que, a pesar de presentar menor efecto anal- gésico, este se ve aumentado por la inhibición en la recaptación de noradrenalina (6-9); por lo que favorece la disminución en la incidencia de efectos adversos y una mayor tolerabilidad al tratamiento en pacientes con dolor crónico neuropático y en otras condiciones, como en la osteoartitis (10) o en cáncer (11). Secundario a su efectividad en el manejo de dolor neuropático, se ha estudiado su combinación con pregabalina y se ha demostrado su efectividad y tolerabilidad gástrica y en sistema nervioso central, aun con tratamiento opioide previo, en pacientes que han mostrado intolerabilidad a través de diversos efectos adversos $(12,13)$.

La clínica del dolor del Centro Médico Nacional (CMN) 20 de Noviembre del ISSSTE, situado en la Ciudad de México, atiende a una gran población del país con esta patología. Los pacientes son un grupo heterogéneo, que incluye un gran rango de edades, muchos de ellos con comorbilidades distintas y que se presentan con dolor lumbar crónico. Estos acuden a la consulta refiriendo dolor axial o radicular, causa importante de ausentismo laboral e incapacidad. En la presente investigación, el tratamiento de toda la población de pacientes, previo a la llegada a la clínica se basaba exclusivamente en pregabalina y antinflamatorios no esteroideos (AINE). Para propósitos de esta investigación se prescribió tapentadol de acción prolongada, para mejorar el control del DN secundario al SPL. Esto significaría una ventana de oportunidad en el control del dolor y con una menor cantidad de efectos adversos y reducción de polifarmacia.

\section{PACIENTES Y MÉTODOS}

Se realizó un estudio unicéntrico, longitudinal, prospectivo y observacional en el CMN 20 de Noviembre. Se seleccionaron a los pacientes que acudieron a la clínica del dolor a consulta de primera vez con diagnóstico de SPL, referidos de unidades de segundo nivel de diferentes provincias de la República Mexicana, que se encontraban bajo tratamiento con una dosis estandarizada de pregabalina $75 \mathrm{mg}$ cada $24 \mathrm{~h}$, por las noches. Con autorización y consentimiento informado previo, donde se explicó detalladamente todos los probables efectos secundarios del tratamiento, se inició terapia con tapentadol de acción prolongada a 100 o $200 \mathrm{mg}$ vía oral cada 24 horas. En la primera consulta se aplicó como prueba diagnóstica el cuestionario Lanss, con la finalidad 
de determinar si el dolor que presentaban los pacientes era de tipo neuropático, y para evaluar el impacto del tratamiento con tapentadol se aplicó el cuestionario Brief Pain Inventory en su versión en español. Durante cuatro semanas se monitorizó vía telefónica una vez a la semana a los pacientes, para ajuste individual de dosis, para determinar aquella dosis que le brinda mayor alivio del dolor con la menor incidencia de efectos adversos, como cefalea, molestias gastrointestinales, boca seca, cansancio, ansiedad o trastornos del sueño.

Para el análisis estadístico de los datos se tomó en consideración a cada ítem del cuestionario Lanss y del cuestionario Brief Pain Inventory como una variable a estudiar. Los ítem estudiados para el análisis estadístico de la evolución de la percepción del dolor del cuestionario BPI corresponden a las siguientes preguntas:

1. Clasifique su dolor haciendo un círculo alrededor del número que describe la intensidad máxima del dolor sentido en las últimas 24 horas.

2. Clasifique su dolor haciendo un círculo alrededor del número que describe la intensidad mínima del dolor sentido en las últimas 24 horas.

3. Clasifique su dolor haciendo un círculo alrededor del número que describe la intensidad media del dolor sentido en las últimas 24 horas.

4. Clasifique su dolor haciendo un círculo alrededor del número que describe la intensidad de su dolor actual.

Posteriormente, se evaluó la percepción de los pacientes ante el cambio en calidad de vida a través del Brief Pain Inventory, el cual evalúa diferentes áreas de impacto secundario al dolor, donde los ítem corresponden a las siguientes áreas: a) actividad general; b) estado de ánimo; c) deambulación; d) trabajo normal; e) relaciones interpersonales; f] calidad del sueño, y g) capacidad de diversión.

Se comparó la percepción del dolor de los pacientes en la valoración inicial en la clínica del dolor, de forma previa a recibir el tratamiento a base de tapentadol, y 4 semanas después de haber comenzado el tratamiento. Para valorar si la diferencia entre ambas observaciones era significativa se aplicó una prueba de rangos de Friedman.

\section{RESULTADOS}

Se trabajó con una muestra de 30 pacientes, de los cuales 19 fueron mujeres $[63,3 \%$ ) y 11 eran hombres [36,6\%). La edad promedio de los pacientes fue de 49,65 años, con un rango de edades que iba de los 25 a los 65 años de edad, la desviación estándar fue de 9,79 años, lo cual nos indica una muestra heterogénea. Se confirmó en el $100 \%$ de la muestra un diagnóstico de SPL y la presentación de un dolor de tipo neuropático. De nuestra población, 14 de los pacientes no habian presentado consumo previo de opioides $[46,6 \%$, mientras que 16 de los pacientes si habían tenido experiencias con opioides [53,3\%). Se dividió a la población en dos grupos de manera aleatorizada, de tal manera que el primer grupo de 13 pacientes (43,3\%) recibió tapentadol a $100 \mathrm{mg}$ vía oral cada 24 horas y el segundo de 17 pacientes $[56,6 \%$ recibió 200 mg vía oral cada 24 horas por cuatro semanas. En el seguimiento de efectos adversos, 21 pacientes (70 \%) no reportaron presentar reacciones adversas; quedando un $30 \%$ de los pacientes con reacciones: 2 de ellos presentaron constipación y 8 presentaron náuseas. Para el análisis estadístico de los datos se tomó en consideración a cada ítem como una variable a estudiar del cuestionario Brief Pain Inventory y del cuestionario de Lanss, comparando la percepción del dolor de los pacientes previo a recibir el medicamento y cuatro semanas después de llevar el tratamiento a través de la Escala Verbal Análoga del Dolor.

Se comparó la intensidad del dolor de los pacientes previo a recibir el medicamento (PreTest) y 4 semanas después de iniciarlo (PostTest), mostrando una disminución significativa de la percepción del dolor en todas las variables, sin embargo la comparación entre la dosis de $100 \mathrm{mg}$ o de $200 \mathrm{mg}$ de tapentadol no mostraron diferencias estadísticamente significativas en ninguno de los grupos; los resultados se muestran en la Figura 1. Posteriormente, en la evaluación del cambio en calidad de vida a través de diferentes áreas de impacto, mostrando en todas las áreas evaluadas que hay una disminución significativa del impacto del dolor y mejora en la percepción de las áreas de impacto estudiadas, nuevamente sin demostrar diferencias entre una dosis u otra de tapentadol; los resultados se muestran en la Figura 2. Adicionalmente, los pacientes refieren disminución en el ardor en las piernas y la sensación de toques eléctricos, teniendo un impacto en la actividad general y pudiendo realizar un mayor número de activi-

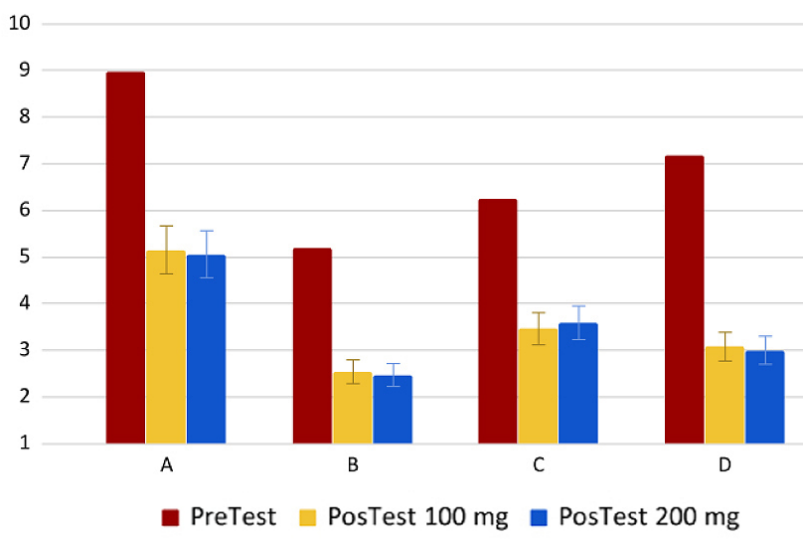

Fig. 1. Percepción de intensidad del dolor previo y posterior al uso de tapentadol. Se muestra el promedio de las calificaciones globales que asignaron los pacientes a la intensidad de su dolor en el comparando la percepción del dolor neuropático previo al tratamiento con tapentadol (PreTest) y posterior al tratamiento (PosTest); adicionalmente se comparan los resultados que obtuvieron el grupo con dosis de $100 \mathrm{mg}$ cada 24 horas contra el grupo de $200 \mathrm{mg}$ cada 24 horas. Las preguntas se grafican y corresponden a las siguientes preguntas: A. Clasifique su dolor haciendo un círculo alrededor del número que describe la intensidad máxima del dolor sentido en las últimas 24 horas; B. Clasifique su dolor haciendo un círculo alrededor del número que describe la intensidad mínima del dolor sentido en las últimas 24 horas; C. Clasifique su dolor haciendo un círculo alrededor del número que describe la intensidad media del dolor sentido en las últimas 24 horas y D. Clasifique su dolor haciendo un círculo alrededor del número que describe la intensidad de su dolor actual. 


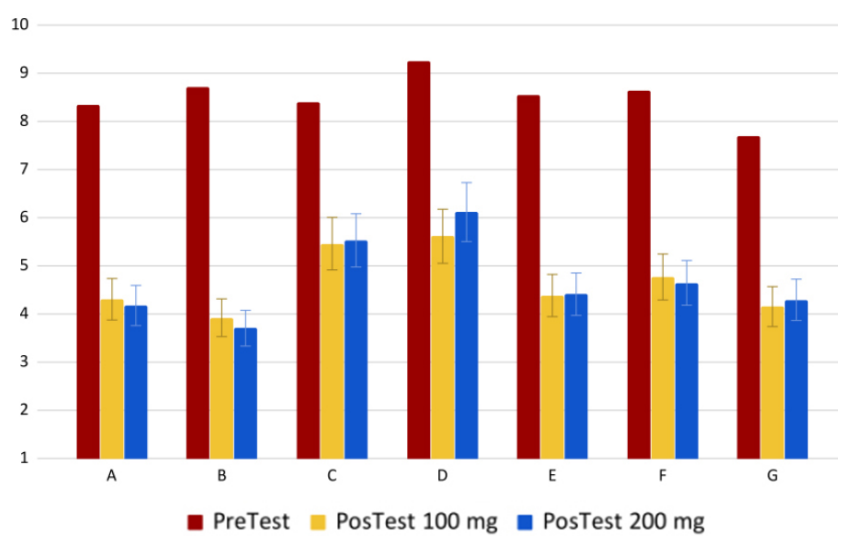

Fig. 2. Impacto del dolor en la vida de los pacientes previo y posterior al uso de tapentadol. Se muestra el promedio de las calificaciones globales que asignaron los pacientes a la afección de distintas áreas de su vida cotidiana y se compara la calificación previa al tratamiento con tapentadol (PreTest) y posterior al tratamiento (PostTest); adicionalmente se comparan los resultados que obtuvieron el grupo con dosis de 100 mg cada 24 horas contra el grupo de 200 mg cada 24 horas. Se aprecia una mejoría significativa de la afección del dolor en la calidad de vida posterior al uso de tapentadol de liberación prolongada, pero no hay diferencia significativa entre el uso de una dosis u otra. Las áreas se grafican de la siguiente manera: A. Actividad general; B. Estado de ánimo; C. Deambulación; D. Trabajo normal; E. Relaciones interpersonales; F. Calidad del sueño, y G. Capacidad de diversión.

dades como caminar, realizar trabajos domésticos, una mejoría en su relación con los otros y, de igual manera, existe una mejoría con relación al sueño.

En relación con los efectos adversos presentados, solo el $25 \%$ refirió náusea, la cual fue transitoria y no llevó a los pacientes a suspender el tratamiento. El $6 \%$ de la muestra refirió constipación, sin embargo, no fue tan intensa como para que el paciente suspendiera el tratamiento.

\section{DISCUSIÓN}

La inhibición de la recaptación de la noradrenalina en los axones terminales de la vía descendente inhibitoria noradrenérgica aumenta la concentración extracelular de noradrenalina a nivel medular, produciendo antinocicepción mediante la activación de los receptores $\alpha 2$ adrenérgicos; se conoce que este hecho es especialmente relevante en los estados de dolor crónico, especialmente en el dolor crónico neuropático $[7,8]$.

Con base en la investigación efectuada, podemos afirmar que el medicamento tapentadol de liberación prolongada es efectivo para el control del DN secundario al SPL, ya que los pacientes estudiados presentan una disminución en la percepción del dolor máximo y la media del dolor de acuerdo a la Escala Verbal Análoga del Dolor, utilizando el cuestionario Brief Pain Inventory en su versión en español y el cuestionario de Lanss, así como una mejoría estadísticamente significativa en evaluación de calidad de vida a través de diferentes áreas de impacto del dolor, donde se mostró una prin- cipal mejoría en el estado de ánimo. Sin embargo, no existen diferencias significativas entre una dosis y otra.

Los estudios realizados de tapentadol en cuanto a equipotencia y equianalgesia han sido comparados contra oxicodona, el cual es un opioide fuerte, y en pacientes sin administración previa de opioides los efectos adversos, con gran frecuencia, llevan al paciente a dejar el tratamiento [9]. A partir de estos resultados y de la baja presentación de efectos adversos al tapentadol, podemos suponer que el apego al tratamiento será mejor, en comparación con los previamente utilizados, llevando a un menor consumo de medicamentos y a una menor exposición a efectos adversos bien conocidos del uso crónico de analgésico opioides. La principal limitación de este estudio fue una muestra pequeña la poca disponibilidad de los pacientes para acudir a revisiones de seguimiento por limitaciones de transporte, las cuales fueron parcialmente resueltas a presentar seguimiento por vía telefónica.

\section{CONCLUSIÓN}

El uso terapéutico de tapentadol de acción prolongada demostró superioridad en el control analgésico del dolor neuropático secundario a síndrome postlaminectomía en pacientes con utilización previa de analgésicos no opioides que expresaron persistencia del dolor postquirúrgico y afección a su calidad de vida. Dentro de sus ventajas analgésicas como opioide, su agonismo parcial mu disminuye la incidencia de efectos adversos. Esta característica pudiera hacer que el tapentadol de acción prolongada se convirtiera en un opioide de primera línea en el tratamiento del dolor neuropático debido a su alta tolerabilidad. Además de manera clínica, algunos de los beneficios observados es una percepción máxima del dolor con una disminución estadísticamente significativa y una mejora en la calidad de vida, especialmente en el estado de ánimo. Con base en los resultados obtenidos podemos concluir que el tapentadol es un medicamento eficaz en el tratamiento del dolor neuropático, con el cual se puede iniciar terapia con opioides, siendo efectiva en el control del dolor neuropático secundario al síndrome postlaminectomía.

\section{CONFLICTO DE INTERESES}

Los autores declaran no tener conflicto de intereses alguno.

\section{FINANCIACIÓN}

La presente investigación no ha recibido ayudas específicas provenientes de agencias del sector público, sector comercial o entidades sin ánimo de lucro.

\section{BIBLIOGRAFÍA}

1. Blond S, Mertens P, David R, Roulaud M, Rigoard P. From "mechanical" to "neuropathic" back pain concept in FBSS patients. A systematic review based on factors leading to the chronification of pain (part C). Neurochirurgie. 2015;61(Suppl 1):S45-56. DOI: 10.1016/j.neuchi.2014.11.001. 
2. Al Kaisy A, Pang D, Desai MJ, Pries P, North R, Taylor RS, et al P. Failed back surgery syndrome: who has failed? Neurochirurgie. 2015;61(Suppl 1):S6-S14. DOI: 10.1016/j. neuchi.2014.10.107.

3. Finnerup NB, Haroutounian S, Kamerman P, Baron R, Bennett DLH, Bouhassira D, et al. Neuropathic pain: an updated grading system for research and clinical practice. Pain. 2016;157(8):1599-606. DOI: 10.1097/j. pain.0000000000000492.

4. Bailey JC, Kurklinsky S, Sletten CD, Osborne MD. The Effectiveness of an Intensive Interdisciplinary Pain Rehabilitation Program in the Treatment of Post-Laminectomy Syndrome in Patients Who Have Failed Spinal Cord Stimulation. Pain Med. 2018;19(2):385-92. DOI: 10.1093/pm/pnx060.

5. Tzschentke TM, Jahnel U, Kogel B, Christoph T, Englberger W, De Vry J, et al. Tapentadol hydrochloride: a next-generation, centrally acting analgesic with two mechanisms of action in a single molecule. Drugs Today (Barc). 2009;45(7):48396. DOl: 10.1358/dot.2009.45.7.1395291.

6. Singh DR, Nag K, Shetti AN, Krishnaveni N. Tapentadol hydrochloride: A novel analgesic. Saudi $J$ Anaesth. 2013;7(3):322-6. DOl: 10.4103/1658-354X.115319.

7. Schröder W, Vry JD, Tzschentke TM, Jahnel U, Christoph T. Differential contribution of opioid and noradrenergic mechanisms of tapentadol in rat models of nociceptive and neuropathic pain. Eur J Pain. 2010;14(8):814-21. DOI: 10.1016/j.ejpain.2010.05.005.
8. Hartrick CT, Rozek RJ. Tapentadol in pain management: a $\mu$-opioid receptor agonist and noradrenaline reuptake inhibitor. CNS Drugs. 2011;25(5):359-70. DOI: 10.2165/11589080-000000000-00000.

9. Caputi FF, Nicora M, Simeone R, Candeletti S, Romualdi P. Tapentadol: an analgesic that differs from classic opioids due to its noradrenergic mechanism of action. Minerva Med. 2019;110(1):62-78. DOI: 10.23736/S00264806.18.05909-8.

10. Steigerwald I, Schenk M, Lahne U, Gebuhr P, Falke D, Hoggart B. Effectiveness and tolerability of tapentadol prolonged release compared with prior opioid therapy for the management of severe, chronic osteoarthritis pain. Clin Drug Investig. 2013;33(9):607-19. DOI: 10.1007/s40261-013-0102-0.

11. Kress HG, Coluzzi F. Tapentadol in the management of cancer pain: current evidence and future perspectives. J Pain Res. 2019;12:1553-60. DOI: 10.2147/JPR.S191543.

12. Deeks ED. Tapentadol Prolonged Release: A Review in Pain Management. Drugs. 2018;78(17):1805-16. DOI: 10.1007/s40265-018-1007-2.

13. Baron R, Martin-Mola E, Müller M, Dubois C, Falke D, Steigerwald I. Effectiveness and Safety of Tapentadol Prolonged Release (PR) Versus a Combination of Tapentadol PR and Pregabalin for the Management of Severe, Chronic Low Back Pain With a Neuropathic Component: A Randomized, Double-blind, Phase 3b Study. Pain Pract. 2015;15(5):45570. DOI: 10.1111/papr.12200. 